\title{
A survey and evaluation of bior wavelet based compression techniques
}

Rashid Hussain *

Faculty of Engineering Science and Technology, Hamdard University, Sharae Madinat Al-Hikmah, Karachi 74600, Pakistan

\section{ARTICLE INFO}

\section{Article history:}

Received 14 June 2016

Received in revised form

4 March 2017

Accepted 5 March 2017

Keywords:

Wavelet compression

Computational constraints

Astronomical images

\begin{abstract}
A B S T R A C T
Reconstruction performances of Wavelet for image compressing have been elucidated by various research studies. The purpose of this research is to expound bior Wavelet for the efficient image compression. Wavelet based compression can decompose and reconstruct image into approximate and diagonal details. Mother wavelet refers to a selection of a suitable wavelet function for dilation and translation versions of mother prototype. Many research focus on the selection of most appropriate mother wavelet for image reconstruction. In this research various compression methods investigated on a bior Wavelet. Experimental results show that Adaptively Scanned Wavelet Difference Reduction technique together with bior1.3 has efficient reconstruction capability. Experimental results also show that bior1.3 showed best performance in terms of compression error and compression ratio.
\end{abstract}

(C) 2017 The Authors. Published by IASE. This is an open access article under the CC BY-NC-ND license (http://creativecommons.org/licenses/by-nc-nd/4.0/).

\section{Introduction}

A wavelet is a mathematical function alternative to the short-time Fourier transform, in which signal can be visualized as a short pulse with finite duration like one recorded by a cardiac monitor. Wavelet transform has a variety of applications in digital signal processing and image compression. It is suitable for approximating the functions with spikes characteristics and irregularities.

Wavelet Packet is the implementation of wavelet transform, in which higher numbers of filters are used to decompose and reconstruct the signal. In the wavelet packet decomposition and reconstruction phase both detail and approximation coefficients form a full binary tree. By this virtue, wavelet packet offers better compression of high perceptual images with a less number of bits. On the contrary, in discrete wavelet transform only previous approximation coefficients are decomposed to form binary tree (Gonzalez and Wood, 2002; Daubechies, 1992; Coifman and Wickerhauser, 1992).

Though standard compression techniques can be effective for storage and transmission, they do suffer from major limitation when image has to be sent from far distance. Wavelet packet has been a proven choice for image compression. It can be utilized for effective compressing images with least number of

\footnotetext{
* Corresponding Author.

Email Address: rashid.hussain@hamdard.edu.pk https://doi.org/10.21833/ijaas.2017.05.005

2313-626X/C 2017 The Authors. Published by IASE.

This is an open access article under the CC BY-NC-ND license

(http://creativecommons.org/licenses/by-nc-nd/4.0/)
}

bits, for an efficient transmission over longer distances. In the wavelet based image compression, the selection of mother wavelet is very critical, as it set limits to the reconstruction performance. This research focuses on the reconstruction performance of bior Wavelet for the following compression methods as seen in Table 1.

Some of the recent researches in image compression include; Compressed-domain ship detection on space borne optical image using deep neural network and extreme learning machine; in this research framework, wavelet coefficients from JPEG2000 compressed domain have been combined with the help of deep neural network (DNN) and extreme learning machine algorithms for the detection of Space borne optical imaging (Tang et al., 2015). A 1.5-D multi-channel EEG compression algorithm based on NLSPIHT; in this study, the authors investigated on 1-D Discrete Wavelet Transform (DWT) for EEG compression, which results in less power dissipation and less computational constraints (Xu et al., 2015). Energyefficient low bit rate image compression in wavelet domain for wireless image sensor networks; in this technique, the authors presented Wavelet transform based approximation band algorithm for low bit rate image compression (Phamil and Amutha, 2015). An innovative lossless compression method for discrete-color images; in this paper relative probability of occurrence of Huffman codes and rowcolumn reduction coding were performed on images (Alzahir and Borici, 2015). A 151 dB High Dynamic Range CMOS Image Sensor Chip Architecture with tone mapping compression embedded in-pixel; in 
the proposed framework, in-pixel content-aware adaptive global tone mapping algorithm is studied and tone mapping curve on the compression function of illuminations is calculated using histogram (Vargas et al., 2015). Novel region-based image compression method based on spiking cortical model; in this proposed method the authors exploits irregular segmentation region coding based on spiking cortical model (Zhao and Ma, 2015). An efficient DCT-based image compression system based on Laplacian transparent composite model; in this paper the authors identify outlier coefficients using Laplacian transparent composite model for image compression system (Sun and Yang, 2015). Multiresolution graph Fourier transform for compression of piecewise smooth images; in this research the authors focused on graph Fourier transforms (GFTs) to minimize the total signal representation cost of each pixel (Wei et al., 2015). A fully embedded two-stage coder for hyperspectral near-lossless compression; this research exploited a two stage near-lossless coder for hyperspectral images (Beerten et al., 2015). Learning-based joint super-resolution and deblocking for a highly compressed image; in this paper, the author investigates how to preserve artifacts using the joint super-resolution technique for highly compressed images (Kang et al., 2015). Recently, lossless compression applied on JPEG coded photo collections (Wu et al., 2016).

Table 1: Wavelet based compression techniques

\begin{tabular}{cc}
\hline Techniques & Description \\
\hline EdgeWize Compressed (EWZ) & $\begin{array}{c}\text { EZW is lossy compression algorithm, which is based on the fact that most of the } \\
\text { coefficients produced after transformation are close to zero, while from human } \\
\text { perception point of view, contours and edges contain high frequency } \\
\text { information in the form of non-zero coefficients, which are enough for } \\
\text { representing high quality coding scheme (Shapiro, 1993). }\end{array}$
\end{tabular}

Symmetric Discrete Wavelet Transform (SWT)

Wavelet difference reduction (WDR)

Adaptively Scanned Wavelet Difference Reduction (ASWDR)

Set Partitioning In Hierarchical Trees(SPIHT)

Set Partitioning In Hierarchical Trees 3D for true color images (SPIHT 3RD)

SWT is the linear transformation implemented by performing Discrete Wavelet Transformation on a peroidized symmetric extension of the input signal. A detai treatment is discussed in (Bradley and Brislawn, 1992). It solves the issues of coefficient expansion and border discontinuities. SWT has been proven to enhance image coding performance (Lightstone et al., 1997).

Like SPIHT encoding stages, it performs a sorting and refining pass for each bit plane. WDR has two major benefits. First, it produces stream of bits for transmission over limited bandwidth channels. Second it caters region of interest based capabilities for compressed images (Tian and Wells, 1996; Walker and Nguyen, 2000)

Unlike WDR encoding approach, the ASWDR encoding approach contains varying order position of wavelet coefficients, made it adaptable to the varying features of the image (Walker and Nguyen, 2000).

SPIHT technique yields an embedded stream of bits with minimum mean square error form best reconstructed image at a variable bit rates. It can be used for lossless applications (Said and Pearlman, 1996).

Unlike quad tree structure in SPIHT technique, octal tree structure is used to represent the association among coefficients in different frequency bands (Kim and Pearlman, 1997).

\section{Materials and methods}

The prime objective of this wavelet based compression analysis is to discover the best compromise between good perceptual result and an expectable compression ratio. In this section, the procedure for assessing the reconstruction performance of bior Wavelet for compressing has been investigated. To demonstrate the performance, the schematic of experimental setup is shown in Fig. 1. During the evaluation process, following nine wavelet based compression techniques are tested on astronomical $512 \times 512$ grey tiff (Fig. 2):

\section{EdgeWize Compressed (EWZ)}

2. Symmetric discrete Wavelet Transform (SWT)

3. Wavelet difference reduction (WDR)

4. Adaptively Scanned Wavelet Difference Reduction (ASWDR)

5. Set Partitioning In Hierarchical Trees(SPIHT)
6. Set Partitioning In Hierarchical Trees 3D for true color images (SPIHT 3RD)

7. Sub band thresholding of coefficients and Huffman encoding (LVL_MMC)

8. Global thresholding of coefficients and fixed encoding (GBL_MMC_F)

9. Global thresholding of coefficients and Huffman encoding (GBL_MMC_H)

Bit per pixel ratio and the compression ratio measure the reconstruction performance. The bit per pixel represents the number of bits required to store one pixel of the image. Compression ratio represents compressed image in term of the storage capacity. L2-norm is a conformity term which ensures precision of preliminary course of estimation.

\section{Results and discussion}

A variety of Bior mother wavelets have been exhibited in this investigation. Various other mother 
wavelets can be demonstrated using Symlet (Daubechies, 1992), Coiflet (Beylkin et al., 1991).

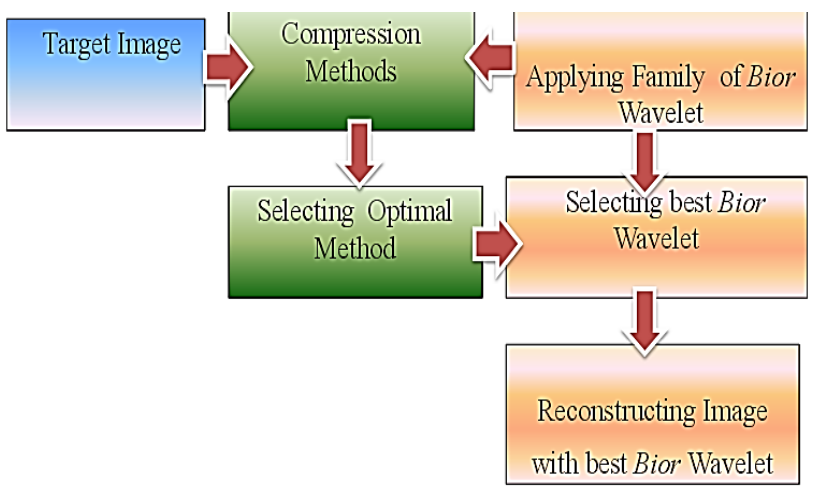

Fig. 1: Schematic describing experimental setup for the reconstruction performance of bior wavelet

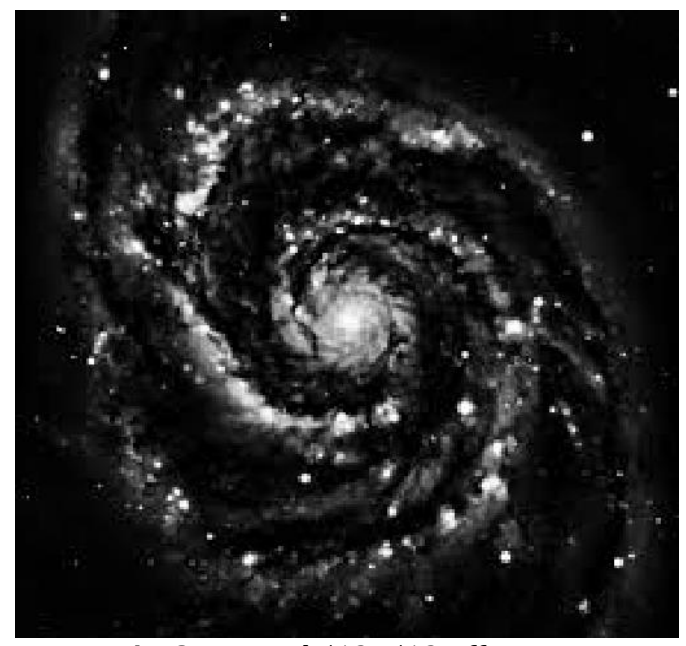

Fig. 2: original 512X512 tiff Image
This implementation has been performed using Matlab based implementation of wavelet based compression methods for bior Wavelet. In this research, the test images went under different sets of analysis. In Table 2, the results for Wavelet based compression methods for bior Wavelet for Fig. 2 are presented.

In the Table 2 and Fig. 3, Wavelet based compression methods for bior Wavelet evaluated for test Fig. 2. For EdgeWize Compressed (EWZ) compression methods, mean square error is 5.391, maximum error is $13 \mathrm{~L}-2$ norm ratio is 99.88, PSNR is 40.81 , bits per pixel is 5.05 and compression ratio is 21.05. For Symmetric discrete Wavelet Transform (SWT) compression methods,, mean square error is 18.5 , maximum error is $25, \mathrm{~L}-2$ norm ratio is 98.89 , PSNR is 53.57 , bits per pixel is 2.82 and compression ratio is 11.75 . For Wavelet difference reduction (WDR) compression methods, mean square error is 7.45, maximum error is 35, L-2 norm ratio is 99.41 , PSNR is 39.4 , bits per pixel is 5.92 and compression ratio is 24.68. For Adaptively Scanned Wavelet Difference Reduction (ASWDR) compression methods,, mean square error is 7.45, maximum error is 35 , L-2 norm ratio is 99.41 , PSNR is 39.4 , bits per pixel is 5.67 and compression ratio is 23.66 . For Set Partitioning In Hierarchical Trees (SPIHT) compression methods, mean square error is 25.01, maximum error is $42, \mathrm{~L}-2$ norm ratio is 98 , PSNR is 43.15 , bits per pixel is 1.84 and compression ratio is 7.67.For Set Partitioning In Hierarchical Trees 3D for truecolor images (SPIHT 3RD) compression methods, mean square error is 25.01, maximum error is 42 , L-2 norm ratio is 98 .

Table 2: Evaluation of wavelet based compression methods for bior wavelet (Fig. 2)

\begin{tabular}{|c|c|c|c|c|c|c|}
\hline Compression Methods & $\begin{array}{c}\text { Mean Square } \\
\text { Error }\end{array}$ & $\begin{array}{l}\text { Maximum } \\
\text { Error }\end{array}$ & $\begin{array}{l}\text { L-2 Norm } \\
\text { Ratio }\end{array}$ & $\begin{array}{c}\text { Peak Signal to } \\
\text { Noise Ratio }\end{array}$ & $\begin{array}{l}\text { Bits per } \\
\text { Pixel } \\
\end{array}$ & $\begin{array}{c}\text { Compression } \\
\text { Ratio } \\
\end{array}$ \\
\hline EdgeWize Compressed (EWZ) & 5.391 & 13 & 99.88 & 40.81 & 5.051 & 21.05 \\
\hline $\begin{array}{c}\text { Symmetric discrete Wavelet } \\
\text { Transform (SWT) }\end{array}$ & 18.5 & 25 & 98.89 & 53.57 & 2.82 & 11.75 \\
\hline Wavelet difference reduction (WDR) & 7.45 & 35 & 99.41 & 39.4 & 5.92 & 24.68 \\
\hline $\begin{array}{l}\text { Adaptively Scanned Wavelet } \\
\text { Difference Reduction (ASWDR) }\end{array}$ & 7.45 & 35 & 99.41 & 39.4 & 5.67 & 23.66 \\
\hline $\begin{array}{c}\text { Set Partitioning In Hierarchical } \\
\text { Trees(SPIHT) }\end{array}$ & 25.01 & 42 & 98 & 43.15 & 1.84 & 7.67 \\
\hline $\begin{array}{l}\text { Set Partitioning In Hierarchical Trees } \\
\text { 3D for truecolor images (SPIHT 3RD) }\end{array}$ & 25.01 & 42 & 98 & 43.15 & 1.03 & 4.44 \\
\hline $\begin{array}{l}\text { Subband thresholding of coefficients } \\
\text { and Huffman encoding (LVL_MMC) }\end{array}$ & 74.53 & 67 & 98.8 & 29.41 & 0.69 & 2.9 \\
\hline $\begin{array}{l}\text { Global thresholding of coefficients and } \\
\text { fixed encoding (GBL_MMC_F) }\end{array}$ & 85.86 & 82 & 99.81 & 28.79 & 0.95 & 4 \\
\hline $\begin{array}{l}\text { Global thresholding of coefficients and } \\
\text { Huffman encoding (GBL_MMC_H) }\end{array}$ & 85.86 & 82 & 99.81 & 28.79 & 0.74 & 3.09 \\
\hline
\end{tabular}

PSNR is 43.15, bits per pixel is 1.03 and compression ratio is 4.44 . For Subband thresholding of coefficients and Huffman encoding (LVL_MMC) compression methods, mean square error is 74.53 , maximum error is $67, \mathrm{~L}-2$ norm ratio is 98.8 , PSNR is 29.41 , bits per pixel is 0.69 and compression ratio is 2.9. For Global thresholding of coefficients and fixed encoding (GBL_MMC_F) compression methods, mean 
square error is 85.86, maximum error is $82, \mathrm{~L}-2$ norm ratio is 98.81, PSNR is 28.79, bits per pixel is 0.95 and compression ratio is 4. For Global thresholding of coefficients and Huffman encoding (GBL_MMC_H) compression methods, mean square error is 85.86 , maximum error is $82, \mathrm{~L}-2$ norm ratio is 98.81 , PSNR is 28.79 , bits per pixel is 0.74 and compression ratio is 3.09 .

\section{Conclusion}

Reconstruction performance of wavelet transforms is crucial for many image processing applications. In contrary to Fourier analysis, where a single set of coefficient is used for describing sine and cosine function, in wavelet analysis, a series of coefficients are used for describing different instants of time. This research has investigated the selection of appropriate wavelet based compression technique together with optimal family. The optimal selection of experimental setup influences the computational constrains for compressing the astronomical images. From image compression analysis, it is concluded that that Adaptively Scanned Wavelet Difference Reduction technique together with bior1.3 has efficient reconstruction capability. Experimental results also show that bior1.3 showed best performance in term of compression error and compression ratio. The summary of the research is as follows:

- In this research, reconstruction performances of wavelet based compression methods have been analyzed. The scope of the reconstruction is to select efficient bior wavelet for computational efficient compression.

- A comparative study has been performed to evaluate the Wavelet based compression methods for bior Wavelet on Fig. 2.

- The performance assessment of the bior Wavelet has been tested using mean square error, maximum error, L-2 norm ratio, peak signal to noise ratio, bits per pixeland compression ratio as shown in shown in Table 2 and in Fig. 3. L2- norm provides the reconstruction performance of compressed image. The visual reconstructed performance is demonstrated through Fig. 4a for original $512 \times 512$ tiff Fig. 2 and Fig. $4 \mathrm{~b}$ for compressed Fig. 2.

- Finally, we reached to the conclusion that bior1.3 Wavelet for ASWDR algorithm showed best performances for low level of decomposition and reconstructions.

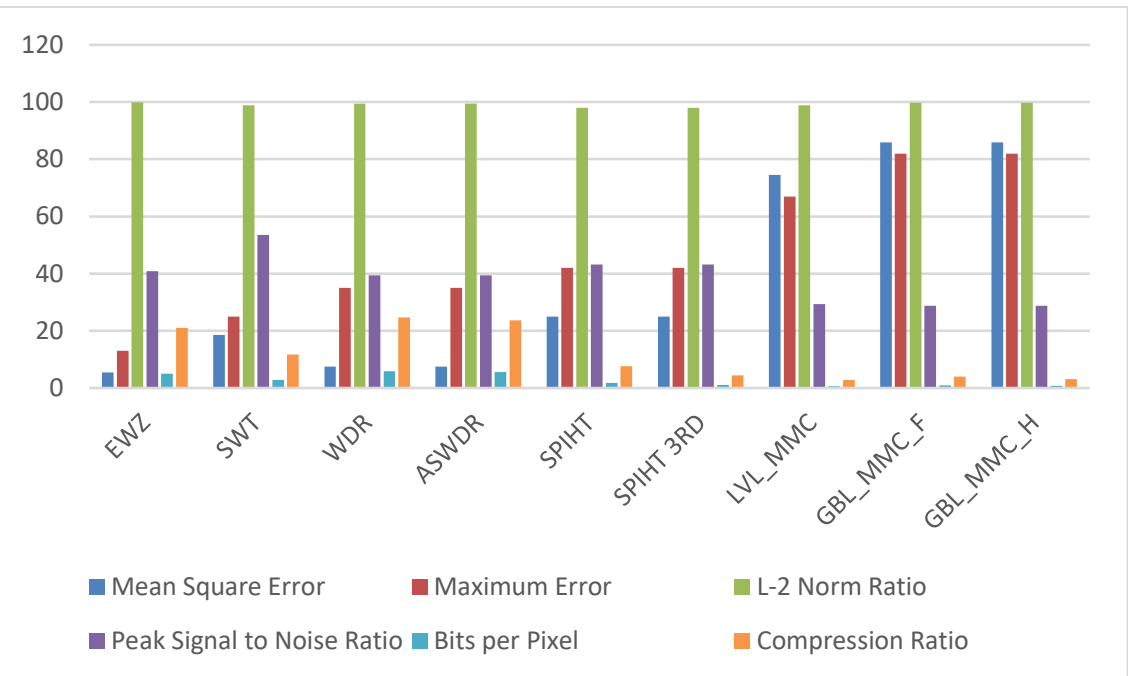

Fig. 3: Evaluation of wavelet based compression methods for bior wavelet on Fig. 2

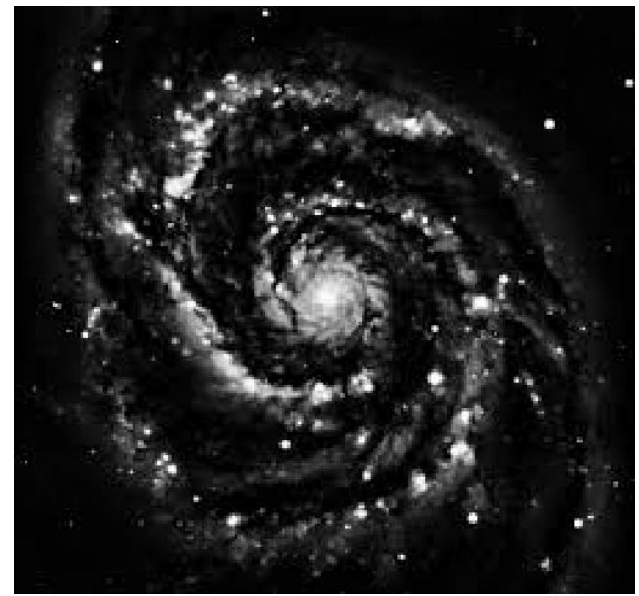

(a)

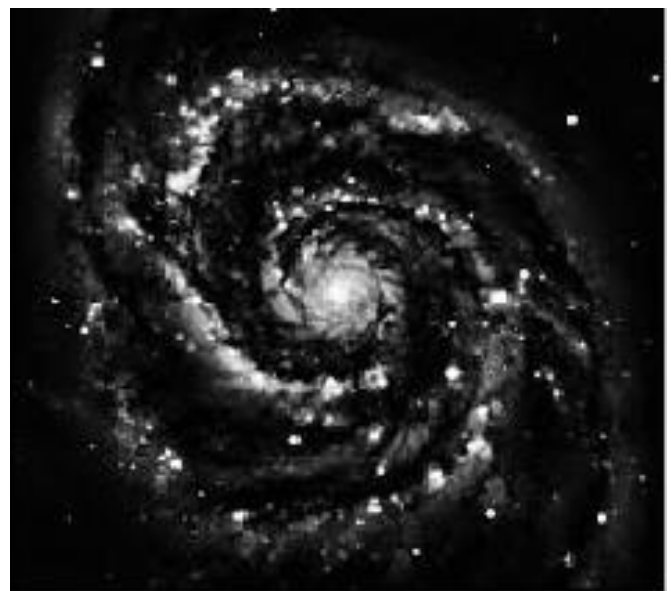

(b)

Fig. 4: Reconstruction is achieved as shown in Fig. 3(a) for original $512 X 512$ tiff Fig. 2 and Fig. 3(b) for compressed Fig. 2 


\section{Acknowledgment}

We would like to thank Gilbert Strang, Mallat S.G., Rafael Gonzalez, and David Donoho for providing a theoretical frame work in image processing. We wish to thank Department of Physics and Astronomy, UCI for providing images. We also wish to thank $\mathrm{M}$. Misiti, Y. Misiti from The MathWorks, Inc for facilitating Matlab based implementation of Wavelet and to the Graduate School of Engineering Sciences and Information Technology, Hamdard University for their logistic support and services.

\section{References}

Alzahir S and Borici A (2015). An innovative lossless compression method for discrete-color images. IEEE Transactions on Image Processing, 24(1): 44-56.

Beerten J, Blanes I, and Serra-Sagristà J (2015). A fully embedded two-stage coder for hyperspectral near-lossless compression. IEEE Geoscience and Remote Sensing Letters, 12(8): 17751779.

Beylkin G, Coifman R, and Rokhlin V (1991). Fast wavelet transforms and numerical algorithms I. Communications on Pure and Applied Mathematics, 44(2): 141-183.

Bradley JN and Brislawn CM (1992). Compression of fingerprint data using the wavelet vector quantization image compression algorithm. Progress Report No. LA-UR--92-1507. Los Alamos National Lab., NM, USA.

Coifman RR and Wickerhauser MV (1992). Entropy-based algorithms for best basis selection. IEEE Transactions on Information Theory, 38(2): 713-718.

Daubechies I (1992). Ten lectures on wavelets. $1^{\text {st }}$ Edition, Society for Industrial and Applied Mathematics, Philadelphia, Pennsylvania, USA.

Gonzalez R and Wood R (2002). Digital image processing. $2^{\text {nd }}$ Edition, Pearson Education Inc., London, England.

Kang LW, Hsu CC, Zhuang B, Lin CW, and Yeh CH (2015). Learningbased joint super-resolution and deblocking for a highly compressed image. IEEE Transactions on Multimedia, 17(7): 921-934.

Kim BJ and Pearlman WA (1997). An embedded wavelet video coder using three dimensional set partitioning in hierarchical trees (3D-SPIHT). In the Proceeding of Data Compression Conference 1997, Snowbird, Utah, USA: 251-260.

Lightstone M, Majani E, and Mitra SK (1997). Low bit-rate design considerations for wavelet-based image coding.
Multidimensional Systems and Signal Processing, 8(1-2): 111128.

Phamil AVY and Amutha R (2015). Energy-efficient low bit rate image compression in wavelet domain for wireless image sensor networks. Browse Journals and Magazines Electronics Letters, 51(11): 824-826.

Said A and Pearlman WA (1996). A new, fast, and efficient image codec based on set partitioning in hierarchical trees. IEEE Transactions on Circuits and Systems for Video Technology, 6(3): 243-250.

Shapiro JM (1993). Embedded image coding using zerotrees of wavelet coefficients. IEEE Transactions on Signal Processing, 41(12): 3445-3462.

Sun C and Yang EH (2015). An efficient DCT-based image compression system based on Laplacian transparent composite model. IEEE Transactions on Image Processing, 24(3): 886-900.

Tang J, Deng C, Huang GB, and Zhao B (2015). Compresseddomain ship detection on spaceborne optical image using deep neural network and extreme learning machine. IEEE Transactions on Geoscience and Remote Sensing, 53(3): 11741185.

Tian J and Wells JrRO (1996). A lossy image codec based on index coding. In the $6^{\text {th }}$ Data Compression Conference, IEEE, Snowbird, Utah, USA: 456-468.

Vargas SS, Liñán-Cembrano G, and Rodríguez-Vázquez Á (2015). A $151 \mathrm{~dB}$ high dynamic range CMOS image sensor chip architecture with tone mapping compression embedded inpixel. IEEE Sensors Journal, 15(1): 180-195.

Walker JS and Nguyen TQ (2000). Adaptive scanning methods for wavelet difference reduction in lossy image compression. In the IEEE International Conference on Image Processing, IEEE, Vancouver, Canada 3: 182-185. https://doi.org/10.1109/ ICIP.2000.899325

Wei H, Cheung G, Ortega A, and Au OC (2015). Multiresolution graph fourier transform for compression of piecewise smooth. IEEE Transactions on Image Processing, 24(1): 419-433.

Wu H, Sun X, Yang J, Zeng W, and Wu F (2016). Lossless compression of JPEG coded Photo collections. IEEE Transactions on Image Processing, 25(6): 2684-2696.

Xu G, Han J, Zou Y, and Zeng X (2015). A 1.5-D multi-channel EEG compression algorithm based on NLSPIHT. IEEE Signal Processing Letters, 22(8): 1118-1122.

Zhao R and Ma Y (2015). Novel region-based image compression method based on spiking cortical model. Journal of Systems Engineering and Electronics, 26(1): 161-171. 